Commun. Fac. Sci. Univ. Ank. Ser. A1 Math. Stat.

Volume 69, Number 1, Pages 613-628(2020)

DOI: $10.31801 /$ cfsuasmas.484452

ISSN 1303-5991 E-ISSN 2618-6470

http://communications.science.ankara.edu.tr/index.php?series=A1

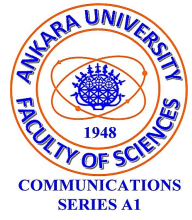

\title{
A GENERALIZED NONLINEAR ITERATIVE ALGORITHM FOR THE EXPLICIT MIDPOINT RULE OF NONEXPANSIVE SEMIGROUP
}

\author{
M. CHERAGHI, M. AZHINI, AND H.R. SAHEBI
}

\begin{abstract}
In this paper, we introduce a new iterative midpoint rule for finding a solution of fixed point problem for a nonexpansive semigroup in real Hilbert spaces. We establish a strong convergence theorem for the sequences generated by our proposed iterative scheme. Furthermore, we provide application to Fredholm integral equations. A numerical example is presented to illustrate the convergence result. Our results improve and extend the corresponding results in the literature.
\end{abstract}

\section{INTRODUCTION}

Let $\mathbb{R}$ denote the set of all real numbers, $H$ be a real Hilbert space with inner product $\langle.,$.$\rangle and norm \|$.$\| and C$ be a nonempty closed convex subset of $H$. A mapping $T: C \rightarrow C$ is said to be contraction if there exists a constant $\alpha \in(0,1)$ such that $\|T(x)-T(y)\| \leq \alpha\|x-y\|$, for all $x, y \in C$. If $\alpha=1, T$ is called nonexpansive on $C$.

The fixed point problem $(F P P)$ for a nonexpansive mapping $T$ is: To find $x \in C$ such that $x \in \operatorname{Fix}(T)$, where Fix $(T)$ is the fixed point set of the nonexpansive mapping $T$.

The explicit midpoint rule is one of the powerful numerical methods for solving ordinary differential equations and differential algebraic equations. For related works, we refer to 2 , 13, 4, 5, 9, 10, 11, 16, 19, 20, 21, 22, 23, 25, 27, 28, and the references cited therein. For instance, consider the initial value problem for the differential equation $y^{\prime}(t)=f(y(t))$ with the initial condition $y(0)=y_{0}$, where $f$ is a continuous function from $\mathbb{R}^{d}$ to $\mathbb{R}^{d}$. The explicit midpoint rule in which a

Received by the editors: November 16, 2018, Accepted: December 08, 2019.

2010 Mathematics Subject Classification. Primary: 47H09, 47H10; Secondary: 47J20.

Key words and phrases. Nonexpansive semigroup, equilibrium problem,midpoint method, strongly positive linear bounded operator, fixed point, Hilbert space.

(C)2020 Ankara University Communications Faculty of Sciences University of Ankara-Series A1 Mathematics and Statistics 
sequence $\left\{y_{n}\right\}$ is generated by the following the recurrence relation

$$
\frac{1}{h}\left(y_{n+1}-y_{n}\right)=f\left(\frac{y_{n+1}-y_{n}}{2}\right) \text {. }
$$

In 2015, Xu et al. 30] extended and generalized the results of Alghamdi et al. [1] and applied the viscosity method on the midpoint rule for nonexpansive mappings and they gave the generalized viscosity explicit method:

$$
x_{n+1}=\alpha_{n} f\left(x_{n}\right)+\beta_{n} x_{n}+\left(1-\alpha_{n}\right) T\left(\frac{x_{n}+x_{n+1}}{2}\right) .
$$

In 2016, Rizvi [24] introduced the following iterative method for the explicit midpoint rule of nonexpansive mappings:

$$
x_{n+1}=\alpha_{n} \gamma f\left(x_{n}\right)+\left(1-\alpha_{n} B\right) T\left(\frac{x_{n}+x_{n+1}}{2}\right) .
$$

A family $S:=\{T(s): 0 \leq s<\infty\}$ of mappings from $C$ into itself is called a nonexpansive semigroup on $C$ if it satisfies the following conditions:

(1) $T(0) x=x$ for all $x \in C$

(2) $T(s+t)=T(s) T(t)$ for all $s, t \geq 0$

(3) $\|T(s) x-T(s) y\| \leq\|x-y\|$ for all $x, y \in C$ and $s \geq 0$

(4) For all $x \in C, s \rightarrow T(s) x$ is continuous.

Plubtieng and Punpaeng [18 introduced and studied the following iterative method to prove a strong convergence theorem for FPP in a real Hilbert space:

$$
x_{n+1}=\alpha_{n} f\left(x_{n}\right)+\beta_{n} x_{n}+\left(1-\alpha_{n}-\beta_{n}\right) \frac{1}{s_{n}} \int_{0}^{s_{n}} T(s) x_{n} d s, \quad \forall n \in \mathbb{N} .
$$

where $f$ is a contraction mapping and $\left\{\alpha_{n}\right\}$ and $\left\{\beta_{n}\right\}$ are the sequences in $(0,1)$ and $\left\{s_{n}\right\}$ is a positive real divergent sequence.

Kang et al. 12] considerd an iterative algorithm in a Hilbert space as follows:

$$
x_{n+1}=\alpha_{n} \gamma f\left(x_{n}\right)+\beta_{n} x_{n}+\left(\left(1-\beta_{n}\right) I-\alpha_{n} A\right) \frac{1}{s_{n}} \int_{0}^{s_{n}} T(s) x_{n} d s .
$$

Under the certain conditions, the sequence $\left\{x_{n}\right\}$ strongly converges to a unique solution of the variational inequality $\left\langle(\gamma f-A) x^{*}, x-x^{*}\right\rangle \leq 0, \forall x \in \operatorname{Fix}(T)$.

Motivated and inspired by the results mentioned and related literature in [1, 12, 24, 30, we propose an iterative midpoint algorithm based on the viscosity method for finding a common element of the set of solutions of nonexpansive semigroup in Hilbert spaces. Then we prove strong convergence theorems that extend and improve the corresponding results of Rizvi [24], Xu [30, and others. Finally, we give an example and numerical result to illustrate our main result.

The rest of paper is organized as follows. The next section presents some preliminary results. Section 3 is devoted to introduce midpoint algorithm for solving it. The last section presents a numerical example to demonstrate the proposed algorithms. 


\section{Preliminaries}

For each point $x \in H$, there exists a unique nearest point of $C$, denote by $P_{C} x$, such that $\left\|x-P_{C} x\right\| \leq\|x-y\|$ for all $y \in C$. $P_{C}$ is called the metric projection of $H$ onto $C$. It is well known that $P_{C}$ is nonexpansive mapping and is characterized by the following property:

$$
\left\langle x-P_{C} x, y-P_{C} y\right\rangle \leq 0
$$

Further, it is well known that every nonexpansive operator $T: H \rightarrow H$ satisfies, for all $(x, y) \in H \times H$, inequality

$$
\langle(x-T(x))-(y-T(y)), T(y)-T(x)\rangle \leq\left(\frac{1}{2}\right)\|(T(x)-x)-(T(y)-y)\|^{2},
$$

and therefore, we get, for all $(x, y) \in H \times F i x(T)$,

$$
\langle(x-T(x)),(y-T(y))\rangle \leq\left(\frac{1}{2}\right)\|(T(x)-x)\|^{2},
$$

see, e.g. 8 .

It is also known that $H$ satisfies Opial's condition [17, i.e., for any sequence $\left\{x_{n}\right\}$ with $x_{n} \rightarrow x$, the inequality

$$
\liminf _{n \rightarrow \infty}\left\|x_{n}-x\right\|<\liminf _{n \rightarrow \infty}\left\|x_{n}-y\right\|
$$

holds for every $y \in H$ with $y \neq x$.

Lemma 1. 6] The following inequality holds in real space $H$ :

$$
\|x+y\|^{2} \leq\|x\|^{2}+2\langle y, x+y\rangle, \quad \forall x, y \in H .
$$

Definition 2. A mapping $M: C \rightarrow H$ is said to be monotone, if

$$
\langle M x-M y, x-y\rangle \geq 0, \quad \forall x, y \in C .
$$

$M$ is called $\alpha$-inverse-strongly-monotone if there exist a positive real number $\alpha$ such that

$$
\langle M x-M y, x-y\rangle \geq \alpha\|M x-M y\|^{2}, \quad \forall x, y \in C .
$$

Definition 3. A mapping $B: H \rightarrow H$ is said to be strongly positive linear bounded operator, if there exists a constant $\bar{\gamma}>0$ such that $\langle B x, x\rangle \geq \bar{\gamma}\|x\|^{2}, \forall x \in H$.

Lemma 4. 15. Assume that $B$ is a strong positive linear bounded self adjoint operator on a Hilbert space $H$ with coefficient $\bar{\gamma}>0$ and $0<\rho \leq\|B\|^{-1}$. Then $\|I-\rho B\| \leq 1-\rho \bar{\gamma}$.

Lemma 5. 26] Let $C$ be a nonempty bounded closed convex subset of a Hilbert space $H$ and let $S=\{T(s): 0 \leq s<\infty\}$ be a nonexpansive semigroup on $C$. For each $x \in C$ and $t>0$. Then, for any $0 \leq h<\infty$,

$$
\lim _{t \rightarrow \infty} \sup _{x \in C}\left\|\frac{1}{t} \int_{0}^{t} T(s) x d s-T(h)\left(\frac{1}{t} \int_{0}^{t} T(s) x d s\right)\right\|=0 .
$$


Lemma 6. 29] Let $\left\{a_{n}\right\}$ be a sequence of nonnegative real numbers such that $a_{n+1} \leq\left(1-\alpha_{n}\right) a_{n}+\delta_{n}, n \geq 0$ where $\alpha_{n}$ is a sequence in $(0,1)$ and $\delta_{n}$ is a sequence in $\mathbb{R}$ such that

(i) $\sum_{n=1}^{\infty} \alpha_{n}=\infty ; \quad$ (ii) $\lim \sup _{n \rightarrow \infty} \frac{\delta_{n}}{\alpha_{n}} \leq 0 \quad$ or $\quad \Sigma_{n=1}^{\infty} \delta_{n}<\infty$.

Then $\lim _{n \rightarrow \infty} a_{n}=0$.

\section{Nonlinear midpoint algorithm}

In this section, we prove a strong convergence theorem based on the explicit iterative for fixed point of nonexpansive semigroup. We firstly present the following unified algorithm.

Let $S=\{T(s): s \in[0,+\infty)\}$ be a nonexpansive semigroup on $C$ such that Fix $(S) \neq \emptyset$. Also $f: C \rightarrow H$ be a $\alpha$-contraction mapping and $B, D$ be strongly positive bounded linear self adjoint operators on $H$ with coefficients $\bar{\gamma}_{1}, \bar{\gamma}_{2}>0$ such that $0<\gamma<\frac{\bar{\gamma}_{1}}{\alpha}<\gamma+\frac{1}{\alpha}, \bar{\gamma}_{1} \leq\|B\| \leq 1$ and $\|D\|=\bar{\gamma}_{2} \leq 1$.

Algorithm 7. For given $x_{0} \in C$ arbitrary, let the sequence $\left\{x_{n}\right\}$ be generated by: $x_{n+1}=\alpha_{n} \gamma f\left(x_{n}\right)+\beta_{n} D x_{n}+\left(\left(1-\epsilon_{n}\right) I-\beta_{n} D-\alpha_{n} B\right) \frac{1}{s_{n}} \int_{0}^{s_{n}} T(s)\left(\frac{x_{n}+x_{n+1}}{2}\right) d s$.

where $\left\{\alpha_{n}\right\},\left\{\beta_{n}\right\},\left\{\epsilon_{n}\right\}$ are the sequence in $(0,1)$ such that $\epsilon_{n} \leq \alpha_{n}$ and $\left\{s_{n}\right\} \subset$ $[s, \infty)$ with $s>0$ satisfying conditions:

(C1)

$$
\begin{gathered}
\lim _{n \rightarrow \infty} \alpha_{n}=\lim _{n \rightarrow \infty} \beta_{n}=\lim _{n \rightarrow \infty} \epsilon_{n}=0, \Sigma_{n=1}^{\infty} \alpha_{n}=\Sigma_{n=1}^{\infty} \beta_{n}=\infty ; \\
\sum_{n=1}^{\infty}\left|\alpha_{n}-\alpha_{n-1}\right|<\infty \quad \text { or } \quad \lim _{n \rightarrow \infty} \frac{\alpha_{n+1}}{\alpha_{n}}=1 ; \\
\sum_{n=1}^{\infty}\left|\beta_{n}-\beta_{n-1}\right|<\infty \quad \text { or } \quad \lim _{n \rightarrow \infty} \frac{\beta_{n+1}}{\beta_{n}}=1 ; \\
\sum_{n=1}^{\infty}\left|\epsilon_{n}-\epsilon_{n-1}\right|<\infty \quad \text { or } \quad \lim _{n \rightarrow \infty} \frac{\epsilon_{n+1}}{\epsilon_{n}}=1 ;
\end{gathered}
$$

$$
\lim _{n \rightarrow \infty} s_{n}=\infty, \sup _{n \in \mathbb{N}}\left|s_{n+1}-s_{n}\right| \text { is bounded. }
$$

Lemma 8. For any $0<\gamma<\frac{\bar{\gamma}_{1}}{\alpha}<\gamma+\frac{1}{\alpha}$, there exists a unique fixed point for sequence $\left\{x_{n}\right\}$.

Proof. As a matter of fact, for fixed $x \in C$, we can define the sequence $\left\{P_{n}: H \rightarrow\right.$ $H\}$ as follows:

$$
P_{n}(x):=\alpha_{n} \gamma f(x)+\beta_{n} D x+\left(\left(1-\epsilon_{n}\right) I-\beta_{n} D-\alpha_{n} B\right) \frac{1}{s_{n}} \int_{0}^{s_{n}} T(s) x d s, \forall x \in H .
$$


We may assume without loss of generality that $\alpha_{n} \leq\left(1-\epsilon_{n}-\beta_{n}\|D\|\right)\|B\|^{-1}$. Since $B$ and $D$ are linear bounded self adjoint operators, we have $\|B\|=\sup \{|\langle B x, x\rangle|: x \in H,\|x\|=1\}$,

$\|D\|=\sup \{|\langle D x, x\rangle|: x \in H,\|x\|=1\}$ and observe that

$$
\begin{aligned}
\left\langle\left(\left(1-\epsilon_{n}\right) I-\beta_{n} D-\alpha_{n} B\right) x, x\right\rangle & =\left(1-\epsilon_{n}\right)\langle x, x\rangle-\beta_{n}\langle D x, x\rangle-\alpha_{n}\langle B x, x\rangle \\
& \geq 1-\epsilon_{n}-\beta_{n}\|D\|-\alpha_{n}\|B\| \geq 0 .
\end{aligned}
$$

Therefore, $\left(1-\epsilon_{n}\right) I-\beta_{n} D-\alpha_{n} B$ is positive. Then, by strong positivity of $B$ and $D$, we get

$$
\begin{aligned}
\left\|\left(1-\epsilon_{n}\right) I-\beta_{n} D-\alpha_{n} B\right\|= & \sup \left\{\left\langle\left(\left(1-\epsilon_{n}\right) I-\beta_{n} D-\alpha_{n} B\right) x, x\right\rangle: x \in H,\|x\|=1\right\} \\
= & \sup \left\{\left(1-\epsilon_{n}\right)\langle x, x\rangle-\beta_{n}\langle D x, x\rangle\right. \\
& \left.-\alpha_{n}\langle B x, x\rangle: x \in H,\|x\|=1\right\} \\
\leq & 1-\epsilon_{n}-\beta_{n} \bar{\gamma}_{2}-\alpha_{n} \bar{\gamma}_{1} \\
\leq & 1-\beta_{n} \bar{\gamma}_{2}-\alpha_{n} \bar{\gamma}_{1} .
\end{aligned}
$$

For any $x, y \in C$

$$
\begin{aligned}
\left\|P_{n} x-P_{n} y\right\| \leq & \alpha_{n} \gamma\|f(x)-f(y)\|+\beta_{n}\|D\|\|x-y\| \\
& +\left\|\left(1-\epsilon_{n}\right) I-\beta_{n} D-\alpha_{n} B\right\| \frac{1}{s_{n}} \int_{0}^{s_{n}}\|T(s) x-T(s) y\| d s \\
\leq & \alpha_{n} \gamma \alpha\|x-y\|+\beta_{n} \bar{\gamma}_{2}\|x-y\|+\left(1-\beta_{n} \bar{\gamma}_{2}-\alpha_{n} \gamma_{1}\right)\|x-y\| \\
= & \left(1-\left(\gamma_{1}-\gamma \alpha\right) \alpha_{n}\right)\|x-y\| .
\end{aligned}
$$

Therefore, Banach contraction principle guarantees that $P_{n}$ has a unique fixed point in $H$, and so the iteration (5) is well defined.

Lemma 9. Let $p \in F i x(S)$. Then the sequence $\left\{x_{n}\right\}$ generated by Algorithm 7 is bounded.

Proof. Let $p \in F i x(S)$, we obtain

$$
\begin{aligned}
\left\|x_{n+1}-p\right\| \leq & \| \alpha_{n} \gamma f\left(x_{n}\right)+\beta_{n} D x_{n} \\
& +\left(\left(1-\epsilon_{n}\right) I-\beta_{n} D-\alpha_{n} B\right) \frac{1}{s_{n}} \int_{0}^{s_{n}} T(s)\left(\frac{x_{n}+x_{n+1}}{2}\right) d s-p \| \\
\leq & \alpha_{n}\left\|\gamma f\left(x_{n}\right)-B p\right\|+\beta_{n}\left\|D x_{n}-D p\right\|+\epsilon_{n}\|p\| \\
& +\left\|\left(\left(1-\epsilon_{n}\right) I-\beta_{n} D-\alpha_{n} B\right)\right\|\left\|\frac{1}{s_{n}} \int_{0}^{s_{n}} T(s)\left(\frac{x_{n}+x_{n+1}}{2}\right)-T(s) p\right\| d s \\
\leq & \alpha_{n}\left(\left\|\gamma f\left(x_{n}\right)-\gamma f(p)\right\|+\|\gamma f(p)-B p\|\right)+\beta_{n}\left\|D x_{n}-D p\right\|+\epsilon_{n}\|p\| \\
& +\left(1-\beta_{n} \bar{\gamma}_{2}-\alpha_{n} \bar{\gamma}_{1}\right)\left\|\frac{x_{n}+x_{n+1}}{2}-p\right\| \\
\leq & \alpha_{n} \gamma \alpha\left\|x_{n}-p\right\|+\alpha_{n}\|\gamma f(p)-B p\|+\beta_{n} \bar{\gamma}_{2}\left\|x_{n}-p\right\|+\alpha_{n}\|p\| \\
& +\frac{\left(1-\beta_{n} \bar{\gamma}_{2}-\alpha_{n} \bar{\gamma}_{1}\right)}{2}\left(\left\|x_{n}-p\right\|+\left\|x_{n+1}-p\right\|\right) .
\end{aligned}
$$


which implies that

$$
\begin{aligned}
\frac{1+\beta_{n} \bar{\gamma}_{2}+\alpha_{n} \bar{\gamma}_{1}}{2}\left\|x_{n+1}-p\right\| \leq & \left(\alpha_{n} \gamma \alpha+\frac{1+\beta_{n} \bar{\gamma}_{2}-\alpha_{n} \bar{\gamma}_{1}}{2}\right)\left\|x_{n}-p\right\| \\
& +\alpha_{n}(\|\gamma f(p)-B p\|+\|p\|) .
\end{aligned}
$$

Then

$$
\begin{aligned}
\left\|x_{n+1}-p\right\| \leq & \left(1-\frac{2\left(\bar{\gamma}_{1}-\gamma \alpha\right) \alpha_{n}}{1+\beta_{n} \bar{\gamma}_{2}+\alpha_{n} \bar{\gamma}_{1}}\right)\left\|x_{n}-p\right\|+\frac{2 \alpha_{n}\left(\bar{\gamma}_{1}-\gamma \alpha\right)}{1+\beta_{n} \bar{\gamma}_{2}+\alpha_{n} \bar{\gamma}_{1}} \frac{\|\gamma f(p)-B p\|+\|p\|}{\bar{\gamma}_{1}-\gamma \alpha} \\
\leq & \max \left\{\left\|x_{n}-p\right\|, \frac{\|\gamma f(p)-B p\|+\|p\|}{\bar{\gamma}_{1}-\gamma \alpha}\right\} \\
& \vdots \\
\leq & \max \left\{\left\|x_{0}-p\right\|, \frac{\|\gamma f(p)-B p\|+\|p\|}{\bar{\gamma}_{1}-\gamma \alpha}\right\} .
\end{aligned}
$$

Hence $\left\{x_{n}\right\}$ is bounded.

Now, set $t_{n}:=\frac{1}{s_{n}} \int_{0}^{s_{n}} T(s)\left(\frac{x_{n}+x_{n+1}}{2}\right) d s$. Then $\left\{t_{n}\right\}$ and $\left\{f\left(x_{n}\right)\right\}$ are bounded.

Lemma 10. The following properties are satisfying for the Algorithm 7

P1. $\lim _{n \rightarrow \infty}\left\|x_{n+1}-x_{n}\right\|=0$.

P2. $\lim _{n \rightarrow \infty}\left\|x_{n}-t_{n}\right\|=0$.

P3. $\lim _{n \rightarrow \infty}\left\|T(s) t_{n}-t_{n}\right\|=0$.

Lemma 11. In order to prove P1, one can write

$$
\begin{aligned}
\left\|t_{n+1}-t_{n}\right\|= & \left\|\frac{1}{s_{n+1}} \int_{0}^{s_{n+1}} T(s)\left(\frac{x_{n+1}+x_{n+2}}{2}\right) d s-\frac{1}{s_{n}} \int_{0}^{s_{n}} T(s)\left(\frac{x_{n}+x_{n+1}}{2}\right) d s\right\| \\
= & \| \frac{1}{s_{n+1}} \int_{0}^{s_{n+1}}\left(T(s)\left(\frac{x_{n+1}+x_{n+2}}{2}\right)-T(s)\left(\frac{x_{n}+x_{n+1}}{2}\right)\right) d s \\
& +\left(\frac{1}{s_{n+1}}-\frac{1}{s_{n}}\right) \int_{0}^{s_{n}} T(s)\left(\frac{x_{n}+x_{n+1}}{2}\right) d s \\
& +\frac{1}{s_{n+1}} \int_{s_{n}}^{s_{n+1}} T(s)\left(\frac{x_{n}+x_{n+1}}{2}\right) d s \| \\
= & \| \frac{1}{s_{n+1}} \int_{0}^{s_{n+1}}\left(T(s)\left(\frac{x_{n+1}+x_{n+2}}{2}\right)-T(s)\left(\frac{x_{n}+x_{n+1}}{2}\right)\right) d s \\
& +\left(\frac{1}{s_{n+1}}-\frac{1}{s_{n}}\right) \int_{0}^{s_{n}}\left(T(s)\left(\frac{x_{n}+x_{n+1}}{2}\right)-T(s) p\right) d s \\
& +\frac{1}{s_{n+1}} \int_{s_{n}}^{s_{n+1}}\left(T(s)\left(\frac{x_{n}+x_{n+1}}{2}\right)-T(s) p\right) d s \| \\
\leq & \left\|\frac{x_{n+1}+x_{n+2}}{2}-\frac{x_{n}+x_{n+1}}{2}\right\|+\frac{\left|s_{n+1}-s_{n}\right| s_{n}}{s_{n+1} s_{n}}\left\|\frac{x_{n}+x_{n+1}}{2}-p\right\|
\end{aligned}
$$




$$
\begin{aligned}
& +\frac{\left|s_{n+1}-s_{n}\right|}{s_{n+1}}\left\|\frac{x_{n}+x_{n+1}}{2}-p\right\| \frac{1}{2}\left(\left\|x_{n+1}-x_{n}\right\|+\left\|x_{n+2}-x_{n+1}\right\|\right) \\
\leq & +\frac{\left|s_{n+1}-s_{n}\right|}{s_{n+1}}\left(\left\|x_{n}-p\right\|+\left\|x_{n+1}-p\right\|\right) .
\end{aligned}
$$

Next, we show that the sequence $\left\{x_{n}\right\}$ is asymptotically regular, i.e., $\lim _{n \rightarrow \infty} \| x_{n+2}-$ $x_{n+1} \|=0$. By (8) we estimate that

$$
\begin{gathered}
\left\|x_{n+2}-x_{n+1}\right\|=\|\left(\alpha_{n+1} \gamma f\left(x_{n+1}\right)+\beta_{n+1} D x_{n+1}\right. \\
\left.+\left(\left(1-\epsilon_{n+1}\right) I-\beta_{n+1} D-\alpha_{n+1} B\right) \frac{1}{s_{n+1}} \int_{0}^{s_{n+1}} T(s)\left(\frac{x_{n+1}+x_{n+2}}{2}\right) d s\right) \\
-\left(\alpha_{n} \gamma f\left(x_{n}\right)+\beta_{n} D x_{n}+\left(\left(1-\epsilon_{n}\right) I-\beta_{n} D-\alpha_{n} B\right) \frac{1}{s_{n}} \int_{0}^{s_{n}} T(s)\left(\frac{x_{n}+x_{n+1}}{2}\right) d s\right) \| \\
=\|\left(\left(1-\epsilon_{n+1}\right) I-\beta_{n+1} D-\alpha_{n+1} B\right)\left(\frac{1}{s_{n+1}} \int_{0}^{s_{n+1}} T(s)\left(\frac{x_{n+1}+x_{n+2}}{2}\right) d s\right. \\
\left.\quad-\frac{1}{s_{n}} \int_{0}^{s_{n}} T(s)\left(\frac{x_{n}+x_{n+1}}{2}\right) d s\right)+\left(\left(\epsilon_{n}+\beta_{n} D+\alpha_{n} B\right)\right. \\
\left.-\left(\epsilon_{n+1}+\beta_{n+1} D+\alpha_{n+1} B\right)\right) \frac{1}{s_{n}} \int_{0}^{s_{n}} T(s)\left(\frac{x_{n}+x_{n+1}}{2}\right) d s+\left(\alpha_{n+1}-\alpha_{n}\right) \gamma f\left(x_{n}\right) \\
+\alpha_{n+1}\left(\gamma f\left(x_{n+1}\right)-\gamma f\left(x_{n}\right)\right)+\left(\beta_{n+1}-\beta_{n}\right) D x_{n}+\beta_{n+1}\left(D x_{n+1}-D x_{n}\right) \| \\
\leq\left(1-\beta_{n+1} \bar{\gamma}_{2}-\alpha_{n+1} \bar{\gamma}_{1}\right)\left\|t_{n+1}-t_{n}\right\|+\left|\epsilon_{n+1}-\epsilon_{n}\right|\left\|\frac{1}{s_{n}} \int_{0}^{s_{n}} T(s)\left(\frac{x_{n}+x_{n+1}}{2}\right) d s\right\| \\
+M\left|\alpha_{n}-\alpha_{n+1}\right|+N\left|\beta_{n}-\beta_{n+1}\right|+\alpha_{n+1} \gamma\left\|f\left(x_{n+1}\right)-f\left(x_{n}\right)\right\| \\
\leq\left(1-\beta_{n+1} \bar{\gamma}_{2}-\alpha_{n+1} \bar{\gamma}_{1}\right)\left\|t_{n+1}-t_{n}\right\|+\left|\epsilon_{n+1}-\epsilon_{n}\right|\left\|\frac{1}{s_{n}} \int_{0}^{s_{n}} T(s)\left(\frac{x_{n}+x_{n+1}}{2}\right) d s\right\| \\
+M\left|\alpha_{n}-\alpha_{n+1}\right|+N\left|\beta_{n}-\beta_{n+1}\right|+\alpha_{n+1} \gamma \alpha\left\|x_{n+1}-x_{n}\right\| \\
\leq \frac{1-\beta_{n+1} \bar{\gamma}_{2}-\alpha_{n+1} \bar{\gamma}_{1}}{2}\left(\left\|x_{n+1}-x_{n}\right\|+\left\|x_{n+2}-x_{n+1}\right\|\right) \\
+\left(1-\beta_{n+1} \bar{\gamma}_{2}-\alpha_{n+1} \bar{\gamma}_{1}\right) \frac{\left|s_{n+1}-s_{n}\right|}{s_{n+1}}\left(\left\|x_{n}-p\right\|+\left\|x_{n+1}-p\right\|\right)+\left|\epsilon_{n+1}-\epsilon_{n}\right|\left\|t_{n}\right\| \\
+M\left|\alpha_{n}-\alpha_{n+1}\right|+N\left|\beta_{n}-\beta_{n+1}\right|+\alpha_{n+1} \gamma \alpha\left\|x_{n+1}-x_{n}\right\|,
\end{gathered}
$$

where $M:=\sup \left\{\left\|\frac{1}{s_{n}} \int_{0}^{s_{n}} T(s)\left(\frac{x_{n}+x_{n+1}}{2}\right) d s\right\|+\left\|f\left(x_{n}\right)\right\|\right\}$ and $N:=\sup \left\{\left\|\frac{1}{s_{n}} \int_{0}^{s_{n}} T(s)\left(\frac{x_{n}+x_{n+1}}{2}\right) d s\right\|+\left\|x_{n}\right\|\right\}$. Then

$$
\begin{gathered}
\left(1+\alpha_{n+1} \bar{\gamma}_{1}+\beta_{n+1} \bar{\gamma}_{2}\right)\left\|x_{n+2}-x_{n+1}\right\| \leq\left(1-\beta_{n+1} \bar{\gamma}_{2}+\left(2 \alpha \gamma-\bar{\gamma}_{1}\right) \alpha_{n+1}\right)\left\|x_{n+1}-x_{n}\right\| \\
+\left(1-\beta_{n+1} \bar{\gamma}_{2}-\alpha_{n+1} \bar{\gamma}_{1}\right) \frac{2\left|s_{n+1}-s_{n}\right|}{s_{n+1}}\left(\left\|x_{n}-p\right\|\right. \\
\left.+\left\|x_{n+1}-p\right\|\right)+2\left|\epsilon_{n+1}-\epsilon_{n}\right|\left\|t_{n}\right\| \\
+2 M\left|\alpha_{n}-\alpha_{n+1}\right|+2 N\left|\beta_{n}-\beta_{n+1}\right| .
\end{gathered}
$$


Therefore

$$
\begin{gathered}
\left\|x_{n+2}-x_{n+1}\right\| \leq\left(1-\frac{2\left(\beta_{n+1} \bar{\gamma}_{2}+\left(\bar{\gamma}_{1}-\alpha \gamma\right) \alpha_{n+1}\right)}{1+\alpha_{n+1} \bar{\gamma}_{1}+\beta_{n+1} \bar{\gamma}_{2}}\right)\left\|x_{n+1}-x_{n}\right\| \\
+\left(\frac{1-\beta_{n+1} \bar{\gamma}_{2}-\alpha_{n+1} \bar{\gamma}_{1}}{1+\alpha_{n+1} \bar{\gamma}_{1}+\beta_{n+1} \bar{\gamma}_{2}}\right)\left(\frac{2\left|s_{n+1}-s_{n}\right|}{s_{n+1}}\right)\left(\left\|x_{n}-p\right\|+\left\|x_{n+1}-p\right\|\right) \\
+\frac{2}{1+\alpha_{n+1} \bar{\gamma}_{1}+\beta_{n+1} \bar{\gamma}_{2}}\left|\epsilon_{n+1}-\epsilon_{n}\right||| t_{n} \|+\frac{2 M}{1+\alpha_{n+1} \bar{\gamma}_{1}+\beta_{n+1} \bar{\gamma}_{2}}\left|\alpha_{n}-\alpha_{n+1}\right| \\
+\frac{2 N}{1+\alpha_{n+1} \bar{\gamma}_{1}+\beta_{n+1} \bar{\gamma}_{2}}\left|\beta_{n}-\beta_{n+1}\right| .
\end{gathered}
$$

Lemma 6 and (C1)-(C2) implies

$$
\lim _{n \rightarrow \infty}\left\|x_{n+1}-x_{n}\right\|=0 .
$$

And similarly, we have

$$
\lim _{n \rightarrow \infty}\left\|x_{n+2}-x_{n+1}\right\|=0 .
$$

Also by (8), (9), (10) and (C3) we have $\lim _{n \rightarrow \infty}\left\|t_{n+1}-t_{n}\right\|=0$.

In order to prove $\mathrm{P} 2$, one can write

$$
\begin{aligned}
\left\|x_{n}-t_{n}\right\| \leq & \left\|x_{n+1}-x_{n}\right\| \\
& +\left\|\alpha_{n} \gamma f\left(x_{n}\right)+\beta_{n} D x_{n}+\left(\left(1-\epsilon_{n}\right) I-\beta_{n} D-\alpha_{n} B\right) t_{n}-t_{n}\right\| \\
\leq & \left\|x_{n}-x_{n+1}\right\|+\alpha_{n}\left\|\gamma f\left(x_{n}\right)-B t_{n}\right\|+\beta_{n} \bar{\gamma}_{2}\left\|x_{n}-t_{n}\right\|+\epsilon_{n}\left\|t_{n}\right\| .
\end{aligned}
$$

Then

$$
\left(1-\beta_{n} \bar{\gamma}_{2}\right)\left\|x_{n}-t_{n}\right\| \leq\left\|x_{n}-x_{n+1}\right\|+\alpha_{n}\left\|\gamma f\left(x_{n}\right)-B t_{n}\right\|+\epsilon_{n}\left\|t_{n}\right\| .
$$

By (C1) and (9), we obtain

$$
\lim _{n \rightarrow \infty}\left\|x_{n}-t_{n}\right\|=0 .
$$

In order to prove P3, set $K:=\left\{w \in C:\|w-p\| \leq\left\|x_{0}-p\right\|+\frac{1}{\bar{\gamma}_{1}-\gamma \alpha}(\|\gamma f(p)-B p\|+\right.$ $\|p\|)\}$. Then $K$ is a nonempty bounded closed convex subset of $C$ which is $T(s)$ invariant for each $s \in[0,+\infty)$ and contains $\left\{x_{n}\right\}$. So, without loss of generality, we may assume that $S:=\{T(s): s \in[0,+\infty)\}$ is a nonexpansive semigroup on $K$.

$$
\begin{aligned}
\left\|T(s) x_{n}-x_{n}\right\|= & \| T(s) x_{n}-T(s) \frac{1}{s_{n}} \int_{0}^{s_{n}} T(s)\left(\frac{x_{n}+x_{n+1}}{2}\right) d s \\
& +T(s) \frac{1}{s_{n}} \int_{0}^{s_{n}} T(s)\left(\frac{x_{n}+x_{n+1}}{2}\right) d s \\
& -\frac{1}{s_{n}} \int_{0}^{s_{n}} T(s)\left(\frac{x_{n}+x_{n+1}}{2}\right) d s+\frac{1}{s_{n}} \int_{0}^{s_{n}} T(s)\left(\frac{x_{n}+x_{n+1}}{2}\right) d s-x_{n} \| \\
\leq & \left\|T(s) x_{n}-T(s) \frac{1}{s_{n}} \int_{0}^{s_{n}} T(s)\left(\frac{x_{n}+x_{n+1}}{2}\right) d s\right\|
\end{aligned}
$$




$$
\begin{aligned}
& +\left\|T(s) \frac{1}{s_{n}} \int_{0}^{s_{n}} T(s)\left(\frac{x_{n}+x_{n+1}}{2}\right) d s-\frac{1}{s_{n}} \int_{0}^{s_{n}} T(s)\left(\frac{x_{n}+x_{n+1}}{2}\right) d s\right\| \\
& +\left\|\frac{1}{s_{n}} \int_{0}^{s_{n}} T(s)\left(\frac{x_{n}+x_{n+1}}{2}\right) d s-x_{n}\right\| \\
\leq & \left\|x_{n}-\frac{1}{s_{n}} \int_{0}^{s_{n}} T(s)\left(\frac{x_{n}+x_{n+1}}{2}\right) d s\right\| \\
& +\left\|T(s) \frac{1}{s_{n}} \int_{0}^{s_{n}} T(s)\left(\frac{x_{n}+x_{n+1}}{2}\right) d s-\frac{1}{s_{n}} \int_{0}^{s_{n}} T(s)\left(\frac{x_{n}+x_{n+1}}{2}\right) d s\right\| \\
& +\left\|\frac{1}{s_{n}} \int_{0}^{s_{n}} T(s)\left(\frac{x_{n}+x_{n+1}}{2}\right) d s-x_{n}\right\| \\
= & 2\left\|\frac{1}{s_{n}} \int_{0}^{s_{n}} T(s)\left(\frac{x_{n}+x_{n+1}}{2}\right) d s-x_{n}\right\| \\
& +\left\|T(s) \frac{1}{s_{n}} \int_{0}^{s_{n}} T(s)\left(\frac{x_{n}+x_{n+1}}{2}\right) d s-\frac{1}{s_{n}} \int_{0}^{s_{n}} T(s)\left(\frac{x_{n}+x_{n+1}}{2}\right) d s\right\|
\end{aligned}
$$

Since $\frac{x_{n}+x_{n+1}}{2} \in C$, from (11) and Lemma 5, we obtain $\lim _{n \rightarrow \infty}\left\|T(s) x_{n}-x_{n}\right\|=0$. Therefore

$$
\begin{aligned}
\left\|T(s) t_{n}-t_{n}\right\| & \leq\left\|T(s) t_{n}-T(s) x_{n}\right\|+\left\|T(s) x_{n}-x_{n}\right\|+\left\|x_{n}-t_{n}\right\| \\
& \leq\left\|t_{n}-x_{n}\right\|+\left\|T(s) x_{n}-x_{n}\right\|+\left\|x_{n}-t_{n}\right\| .
\end{aligned}
$$

Then we have $\lim _{n \rightarrow \infty}\left\|T(s) t_{n}-t_{n}\right\|=0$.

\section{Convergence Algorithm}

Theorem 12. The Algorithm (5) converges strongly $z \in F i x(S)$, which is a unique solution of the variational inequality $\langle(\gamma f-B) z, y-z\rangle \leq 0$, for all $y \in F i x(S)$.

Proof. Let $q=P_{\text {Fix }(S)}$. We get

$$
\begin{aligned}
\|q(I-B+\gamma f)(x)-q(I-B+\gamma f)(y)\| & \leq\|(I-B+\gamma f)(x)-(I-B+\gamma f)(y)\| \\
& \leq\|I-B\|\|x-y\|+\gamma\|f(x)-f(y)\| \\
& \leq\left(1-\bar{\gamma}_{1}\right)\|x-y\|+\gamma \alpha\|x-y\| \\
& =\left(1-\left(\bar{\gamma}_{1}-\gamma \alpha\right)\right)\|x-y\| .
\end{aligned}
$$

Then $q(I-B+\gamma f)$ is a contraction mapping from $H$ into itself. Therefore by Banach contraction principle, there exists $z \in H$ such that $z=q(I-B+\gamma f) z=$ $P_{F i x(S)}(I-B+\gamma f) z$.

We show that $\left\langle(\gamma f-B) z, x_{n}-z\right\rangle \leq 0$. To show this inequality, we choose a subsequence $\left\{t_{n_{i}}\right\}$ of $\left\{t_{n}\right\}$ such that

$$
\limsup _{n \rightarrow \infty}\left\langle(\gamma f-B) z, t_{n}-z\right\rangle=\lim _{i \rightarrow \infty}\left\langle(\gamma f-B) z, t_{n_{i}}-z\right\rangle .
$$

Since $\left\{t_{n_{i}}\right\}$ is bounded, there exists a subsequence $\left\{t_{n_{i_{j}}}\right\}$ of $\left\{t_{n_{i}}\right\} \subseteq K$ which converges weakly to some $w \in C$. Without loss of generality, we can assume that 
$t_{n_{i}} \rightarrow w$. Now, we prove that $w \in F i x(S)$. Assume that $w \notin F i x(S)$. Since $t_{n_{i}} \rightarrow w$ and $T(s) w \neq w$, from Opial's conditions (4) and Lemma 10 (P3), we have

$$
\begin{aligned}
\liminf _{i \rightarrow \infty}\left\|t_{n_{i}}-w\right\| & <\liminf _{i \rightarrow \infty}\left\|t_{n_{i}}-T(s) w\right\| \\
& \leq \liminf _{i \rightarrow \infty}\left(\left\|t_{n_{i}}-T(s) t_{n_{i}}\right\|+\left\|T(s) t_{n_{i}}-T(s) w\right\|\right) \\
& \leq \liminf _{i \rightarrow \infty}\left\|t_{n_{i}}-w\right\|,
\end{aligned}
$$

which is a contradiction. Thus, we obtain $w \in F i x(S)$. Now from (1), we have

$$
\begin{aligned}
\limsup _{n \rightarrow \infty}\left\langle(\gamma f-B) z, x_{n}-z\right\rangle & =\limsup _{n \rightarrow \infty}\left\langle(\gamma f-B) z, t_{n}-z\right\rangle \\
& \leq \limsup _{i \rightarrow \infty}\left\langle(\gamma f-B) z, t_{n_{i}}-z\right\rangle \\
& =\langle(\gamma f-B) z, w-z\rangle \leq 0 .
\end{aligned}
$$

Now we prove that $x_{n}$ is strongly convergence to $z$.

$$
\begin{aligned}
\left\|x_{n+1}-z\right\|^{2}= & \alpha_{n}\left\langle\gamma f\left(x_{n}\right)-B z, x_{n+1}-z\right\rangle+\beta_{n}\left\langle D x_{n}-D z, x_{n+1}-z\right\rangle \\
& -\epsilon_{n}\left\langle z, x_{n+1}-z\right\rangle+\left\langle\left(\left(1-\epsilon_{n}\right) I-\beta_{n} D-\alpha_{n} B\right)\left(t_{n}-z\right), x_{n+1}-z\right\rangle \\
\leq & \alpha_{n}\left(\gamma\left\langle f\left(x_{n}\right)-f(z), x_{n+1}-z\right\rangle+\left\langle\gamma f(z)-B z, x_{n+1}-z\right\rangle\right) \\
& +\beta_{n}\|D\|\left\|x_{n}-z\right\|\left\|x_{n+1}-z\right\|-\epsilon_{n}\|z\|\left\|x_{n+1}-z\right\| \\
& +\left\|\left(1-\epsilon_{n}\right) I-\beta_{n} D-\alpha_{n} B\right\|\left\|t_{n}-z\right\|\left\|x_{n+1}-z\right\| \\
\leq & \alpha_{n} \alpha \gamma\left\|x_{n}-z\right\|\left\|x_{n+1}-z\right\|+\alpha_{n}\left\langle\gamma f(z)-B z, x_{n+1}-z\right\rangle \\
& +\beta_{n} \bar{\gamma}_{2}\left\|x_{n}-z\right\|\left\|x_{n+1}-z\right\|-\epsilon_{n}\|z\|\left\|x_{n+1}-z\right\| \\
& +\left(1-\beta_{n} \bar{\gamma}_{2}-\alpha_{n} \bar{\gamma}_{1}\right) \| \frac{x_{n}+x_{n+1}-z\|\| x_{n+1}-z \|}{2} \\
\leq & \alpha_{n} \alpha \gamma\left\|x_{n}-z\right\|\left\|x_{n+1}-z\right\|+\alpha_{n}\left\langle\gamma f(z)-B z, x_{n+1}-z\right\rangle \\
& +\beta_{n} \bar{\gamma}_{2}\left\|x_{n}-z\right\|\left\|x_{n+1}-z\right\|-\epsilon_{n}\|z\|\left\|x_{n+1}-z\right\| \\
& +\frac{1-\beta_{n} \bar{\gamma}_{2}-\alpha_{n} \bar{\gamma}_{1}\left(\left\|x_{n}-z\right\|+\left\|x_{n+1}-z\right\|\right)\left\|x_{n+1}-z\right\|}{2} \\
= & \frac{1+\beta_{n} \bar{\gamma}_{2}-\alpha_{n}\left(\bar{\gamma}_{1}-2 \alpha \gamma\right)}{2}\left\|x_{n}-z\right\|\left\|x_{n+1}-z\right\|+\alpha_{n}\left\langle\gamma f(z)-B z, x_{n+1}-z\right\rangle \\
& -\epsilon_{n}\|z\|\left\|x_{n+1}-z\right\|+\frac{1-\beta_{n} \bar{\gamma}_{2}-\alpha_{n} \bar{\gamma}_{1}\left\|x_{n+1}-z\right\|^{2}}{2} \\
\leq & \frac{1+\beta_{n} \bar{\gamma}_{2}-\alpha_{n}\left(\bar{\gamma}_{1}-2 \alpha \gamma\right)}{4}\left(\left\|x_{n}-z\right\|^{2}+\left\|x_{n+1}-z\right\|^{2}\right) \\
& +\alpha_{n}\left\langle\gamma f(z)-B z, x_{n+1}-z\right\rangle-\epsilon_{n}\|z\|\left\|x_{n+1}-z\right\| \\
& +\frac{1-\beta_{n} \bar{\gamma}_{2}-\alpha_{n} \bar{\gamma}_{1}\left\|x_{n+1}-z\right\|^{2}}{2} \\
\leq & \frac{1+\beta_{n} \bar{\gamma}_{2}-\alpha_{n}\left(\bar{\gamma}_{1}-2 \alpha \gamma\right)}{4}\left\|x_{n}-z\right\|^{2} \\
&
\end{aligned}
$$




$$
\begin{aligned}
& +\frac{3-\beta_{n} \bar{\gamma}_{2}-\alpha_{n}\left(3 \bar{\gamma}_{1}-2 \alpha \gamma\right)}{4}\left\|x_{n+1}-z\right\|^{2} \\
& +\alpha_{n}\left\langle\gamma f(z)-B z, x_{n+1}-z\right\rangle-\epsilon_{n}\|z\|\left\|x_{n+1}-z\right\| \\
\leq & \frac{1+\beta_{n} \bar{\gamma}_{2}-\alpha_{n}\left(\bar{\gamma}_{1}-2 \alpha \gamma\right)}{4}\left\|x_{n}-z\right\|^{2}+\frac{3}{4}\left\|x_{n+1}-z\right\|^{2} \\
& +\alpha_{n}\left\langle\gamma f(z)-B z, x_{n+1}-z\right\rangle-\epsilon_{n}\|z\|\left\|x_{n+1}-z\right\| .
\end{aligned}
$$

This implies that

$$
\begin{aligned}
4\left\|x_{n+1}-z\right\|^{2} \leq & \left(1+\beta_{n} \bar{\gamma}_{2}-\alpha_{n}\left(\bar{\gamma}_{1}-2 \alpha \gamma\right)\right)\left\|x_{n}-z\right\|^{2}+3\left\|x_{n+1}-z\right\|^{2} \\
& +4 \alpha_{n}\left\langle\gamma f(z)-B z, x_{n+1}-z\right\rangle+4 \epsilon_{n}\|z\|\left\|x_{n+1}-z\right\| .
\end{aligned}
$$

Then

$$
\begin{aligned}
\left\|x_{n+1}-z\right\|^{2} \leq & \left(1-\left(\alpha_{n}\left(\bar{\gamma}_{1}-2 \alpha \gamma\right)-\beta_{n} \bar{\gamma}_{2}\right)\right)\left\|x_{n}-z\right\|^{2} \\
& +4 \alpha_{n}\left\langle\gamma f(z)-B z, x_{n+1}-z\right\rangle+4 \epsilon_{n}\|z\|\left\|x_{n+1}-z\right\| \\
= & \left(1-k_{n}\right)\left\|x_{n}-z\right\|^{2}+4 \alpha_{n} l_{n},
\end{aligned}
$$

where $k_{n}=\alpha_{n}\left(\bar{\gamma}_{1}-2 \alpha \gamma\right)+\beta_{n} \bar{\gamma}_{2}$ and $l_{n}=\left\langle\gamma f(z)-B z, x_{n+1}-z\right\rangle-\|z\|\left\|x_{n+1}-z\right\|$. Since $\lim _{n \rightarrow \infty} \alpha_{n}=\lim _{n \rightarrow \infty} \beta_{n}=0$ and $\Sigma_{n=0}^{\infty} \alpha_{n}=\Sigma_{n=0}^{\infty} \beta_{n}=\infty$, it is easy to see that $\lim _{n \rightarrow \infty} k_{n}=0, \Sigma_{n=0}^{\infty} k_{n}=\infty$ and $\lim \sup _{n \rightarrow \infty} l_{n} \leq 0$. Hence, from (13) and (14) and Lemma 6, we deduce that $x_{n} \rightarrow z$, where $z=P_{F i x(S)}(I-B+\gamma f) z$.

\section{Numerical EXAMPLES}

In this section, we give some examples and numerical results for supporting our main theorem. All the numerical results have been produced in Matlab 2017 on a Linux workstation with a $3.8 \mathrm{GHZ}$ Intel annex processor and $8 \mathrm{~Gb}$ of memory

Example 13. Consider a Fredholm integral equation of the following form

$$
x(t)=g(t)+\int_{0}^{t} F(t, k, x(k)) \quad d k, \quad t \in[0,1],
$$

where $g$ is a continuous function on $[0,1]$ and $F:[0,1] \times[0,1] \times R \rightarrow R$ is continuous. Note that if $F$ satisfies the Lipschitz continuity condition, i.e.,

$$
|F(t, k, x)-F(t, k, y)| \leq|x-y|, \quad \forall t, k \in[0,1], \quad x, y \in \mathbb{R},
$$

then equation (15) has at least one solution in $L^{2}[0,1]$ (see [13]).

Define a mapping $T(s): L^{2}[0,1] \rightarrow L^{2}[0,1]$ by

$$
(T(s) x)(t)=e^{-3 s}\left(g(t)+\int_{0}^{t} F(t, k, x(k)) d k\right), \quad t \in[0,1] .
$$

It is easy to observe that $S=\{T(s): s \in[0,+\infty)\}$ is a nonexpansive semigroup. In fact, we have, for $x, y \in L^{2}[0,1]$,

$$
\|T(s) x-T(s) y\|^{2}=\int_{0}^{1}|(T(s) x)(t)-(T(s) y)(t)|^{2} d t
$$




$$
\begin{aligned}
& =\int_{0}^{1}\left|e^{-3 s} \int_{0}^{1}(F(t, k, x(k))-F(t, k, y(k))) d k\right|^{2} d t \\
& \leq \int_{0}^{1}\left(\int_{0}^{1}|x(k)-y(k)|^{2} d k\right) d t \\
& =\int_{0}^{1}|x(k)-y(k)|^{2} d k \\
& =\|x-y\|^{2} .
\end{aligned}
$$

This means that to find the solution of integral equation (15) is reduced to find a fixed point of the nonexpansive semigroup $S$ in $L^{2}[0,1]$. For any given function $x_{0} \in L^{2}[0,1]$, define a sequence of functions $x_{n}$ in $L^{2}[0,1]$ by

$x_{n+1}=\alpha_{n} \gamma f\left(x_{n}\right)+\beta_{n} D x_{n}+\left(\left(1-\epsilon_{n}\right) I-\beta_{n} D-\alpha_{n} B\right) \frac{1}{s_{n}} \int_{0}^{s_{n}} T(s)\left(\frac{x_{n}+x_{n+1}}{2}\right) d s$

satisfying the conditions of Algorithm 7 . Then the sequence $\left\{x_{n}\right\}$ converges strongly in $L^{2}[0,1]$ to the solution of integral equation (15) which is also a solution of the following variational inequality

$$
\langle(\gamma f-B) z, y-z\rangle \leq 0, \quad \forall y \in F i x(S) .
$$

Example 14. Let $H=R$, the set of all real numbers, with the inner product defined by $\langle x, y\rangle=x y, \forall x, y \in R$, and induced usual norm $|$.$| . Let C=[-1,3]$; Let $f(x)=\frac{1}{9} x, B(x)=\frac{1}{4} x, D(x)=x$ and let, for each $x \in C, T(s) x=\frac{1}{1+2 s} x$. Then there exists a unique sequence $\left\{x_{n}\right\} \subset R$ generated by the iterative scheme

$$
\begin{aligned}
x_{n+1}= & \left(\frac{1}{9 \sqrt{n}}+\frac{1}{2 n}\right) x_{n} \\
& +\left(\left(1-\frac{1}{(n+1)^{2}}\right) I-\frac{1}{2 n} D-\frac{1}{\sqrt{n}} B\right) \frac{1}{s_{n}} \int_{0}^{s_{n}} \frac{1}{1+2 s}\left(\frac{x_{n}+x_{n+1}}{2}\right) d s
\end{aligned}
$$

where $\alpha_{n}=\frac{1}{\sqrt{n}}, \beta_{n}=\frac{1}{2 n}, \epsilon_{n}=\frac{1}{(n+1)^{2}}$ and $s_{n}=n$. Then $\left\{x_{n}\right\}$ converges to $\{0\} \in F i x(S)$. $f$ is contraction mapping with constant $\alpha=\frac{1}{6}$ and $B, D$ are strongly positive bounded linear operators with constant $\bar{\gamma}_{1}=\frac{1}{5}$ on $C$. Therefore, we can choose $\gamma=1$ which satisfies $0<\gamma<\frac{\bar{\gamma}}{\alpha}<\gamma+\frac{1}{\alpha}$. Furthermore, it is easy to observe that Fix $(S)=\{0\} \neq \emptyset$. After simplification, scheme (16) reduce to

$$
x_{n+1}=\frac{\frac{1}{9 \sqrt{n}}+\frac{1}{2 n}+\frac{1}{4 n}\left(1-\frac{1}{(n+1)^{2}}-\frac{1}{2 n}-\frac{1}{4 \sqrt{n}}\right) \ln (1+2 n)}{1-\frac{1}{4 n}\left(1-\frac{1}{(n+1)^{2}}-\frac{1}{2 n}-\frac{1}{4 \sqrt{n}}\right) \ln (1+2 n)} x_{n} .
$$

Following the proof of Theorem 12, we obtain that $\left\{x_{n}\right\}$ converges strongly to $w=$ $\{0\} \in \operatorname{Fix}(S)$. 


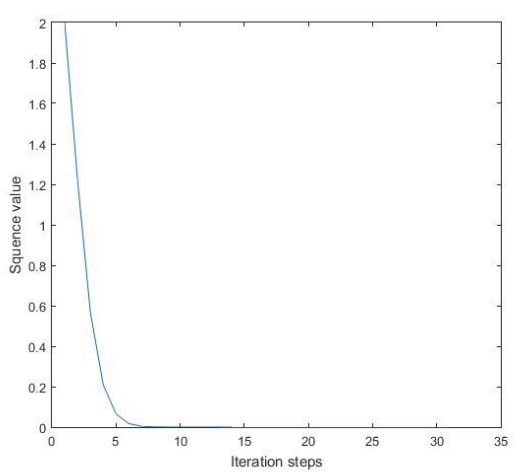

The graph of $x(n)$ with initial value $x(1)=2$

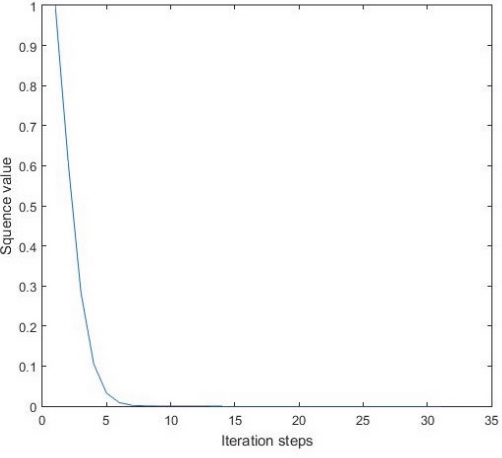

The graph of $x(n)$ with initial value $x(1)=1$

Let $H=R$, the set of all real numbers, with the inner product defined by $\langle x, y\rangle=$ $x y, \forall x, y \in R$, and induced usual norm $|$.$| . Let C=[0,4]$; Let $f(x)=\frac{1}{10}(x-$ $3), B(x)=\frac{1}{3} x, D(x)=\frac{1}{2} x$ and let, for each $x \in C, T(s) x=e^{-2 s} x$. Then there exists a unique sequence $\left\{x_{n}\right\} \subset R$ generated by the iterative scheme

$$
\begin{aligned}
x_{n+1}= & \frac{3}{20 n+5}\left(x_{n}-3\right)+\frac{1}{2 \sqrt{n+2}} x_{n} \\
& +\left(\left(1-\frac{1}{n^{2}}\right) I-\frac{1}{\sqrt{n+2}} D-\frac{3}{4 n+1} B\right) \frac{1}{s_{n}} \int_{0}^{s_{n}} e^{-2 s}\left(\frac{x_{n}+x_{n+1}}{2}\right) d s
\end{aligned}
$$

where $\alpha_{n}=\frac{3}{4 n+1}, \beta_{n}=\frac{1}{\sqrt{n+2}}, \epsilon_{n}=\frac{1}{n^{2}}$ and $s_{n}=2 n$. Then $\left\{x_{n}\right\}$ converges to $\{0\} \in F i x(S)$. $f$ is contraction mapping with constant $\alpha=\frac{1}{9}$ and $B, D$ are strongly positive bounded linear operators with constant $\bar{\gamma}_{1}=\frac{1}{4}$ on $C$. Therefore, we can choose $\gamma=2$ which satisfies $0<\gamma<\frac{\bar{\gamma}}{\alpha}<\gamma+\frac{1}{\alpha}$. Furthermore, it is easy to observe that $\operatorname{Fix}(S)=\{0\} \neq \emptyset$. After simplification, scheme (17) reduce to

$$
x_{n+1}=\frac{\left(\frac{3}{20 n+5}+\frac{1}{2 \sqrt{n+2}}-\frac{1}{8 n}\left(e^{-4 n}-1\right)\left(1-\frac{1}{n^{2}}-\frac{1}{2 \sqrt{n+2}}-\frac{1}{4 n+1}\right)\right) x_{n}-\frac{9}{20 n+5}}{1+\frac{1}{8 n}\left(e^{-4 n}-1\right)\left(1-\frac{1}{n^{2}}-\frac{1}{2 \sqrt{n+2}}-\frac{1}{4 n+1}\right)} .
$$

Following the proof of Theorem 12, we obtain that $\left\{x_{n}\right\}$ converges strongly to $w=$ $\{0\} \in F i x(S)$. 


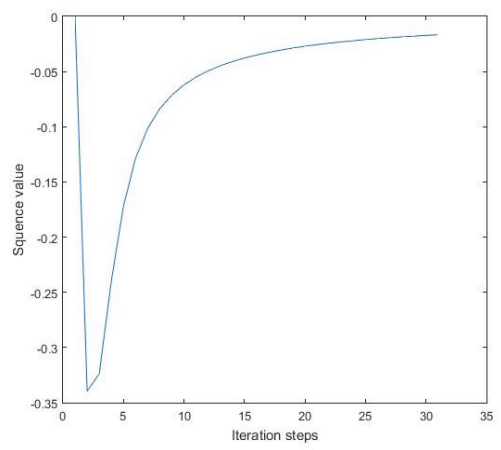

The graph of $x(n)$ with intial value $x(1)=0$

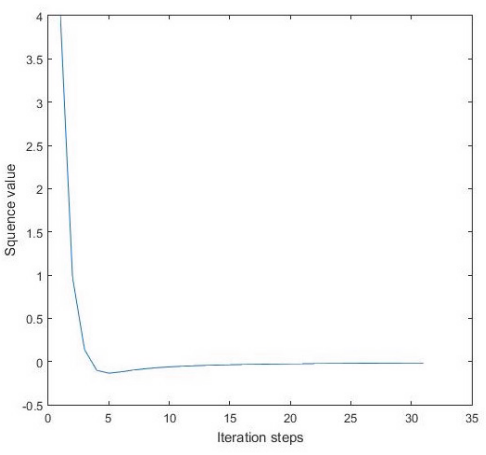

The graph of $x(n)$ with inital value $x(1)=4$

\section{Conculsion}

In this paper, we present a viscosity nonlinear midpoint algorithm for solving equilibrium problems in real Hilbert spaces. The methods propose a theoretical generalization of some existing results in the literature and primary numerical experiments also demonstrate the potential applicability of these methods. We establish the algorithm's strong convergence under mild and standard assumptions. This work open the doors for many promising research directions such as obtaining error bound and convergence rate of our algorithms as well as extensions to Banach spaces.

\section{REFERENCES}

[1] Alghamdi, M.A., Shahzad, N. and Xu, H.K., The implicit midpoint rule for nonexpansive mappings, Fixed Point Theory Appl., 96 (2014), 9 pages.

[2] Auzinger, W. and Frank, R., Asymptotic error expansions for stiff equations: an analysis for the implicit midpoint and trapezoidal rules in the strongly stiff case, Numer. Math., 56 (1989) 469-499.

[3] Bader, G. and Deuflhard, P., A semi-implicit mid-point rule for stiff systems of ordinary differential equations, Numer. Math., 41 (1983) 373-398.

[4] Bagherboum, M. and Razani, A. A., modified Mann iterative scheme for a sequence of nonexpansive mappings and a monotone mapping with applications, Bulletin of the Iranian Mathematical Society, 40 (2014), No. 4, 823-849

[5] Bayreuth, A. The implicit midpoint rule applied to discontinuous differential equations, Computing, 49 (1992) 45-62.

[6] Chang, S. S., Lee, J. and Chan, H. W., An new method for solving equilibrium problem, fixed point problem and variational inequality problem with application to optimization, Nonlinear Analysis, 70(2009)3307-3319.

[7] Chen, R. and Song, Y., Convergence to common fixed point of nonexpansive semigroups, J. Comput. Appl. Math. 200, 566-575 (2007).

[8] Crombez, G., A hicrarchical presentation of operators with fixed points on Hilbert spaces, Numer. Funct. Anal. Optim. 27(2006)259-277. 
[9] Deuflhard, P., Recent progress in extrapolation methods for ordinary differential equations, SIAM Rev., 27(4) (1985) 505-535.

[10] Hofer, E., A partially implicit method for large stiff systems of ODEs with only few equations introducing small time-constants, SIAM J. Numer. Anal., 13 (1976) 645-663.

[11] Homaeipour, S. and Razani, A., Convergence of an iterative method for relatively nonexpansive multi-valued mappings and equilibrium problems in Banach spaces, Optimization Letters, 8 (2014), no. 1, 211-225.

[12] Kang, J., Su, Y. and Zhang, X., Genaral iterative algorithm for nonexpansive semigroups and variational inequalitis in Hilbert space, Journal of Inequalities and Applications (2010) Article ID.264052, 10 pages.

[13] Lions, P.L., Approximation de points fixes de contractions, C.R. Acad. Sci., Ser. A-B, Paris, 284 (1977) 1357-1359.

[14] Mahdioui, H. and Chadli, O., On a system of generalized mixed equilibrium problem involving variational-like inequalities in Banach spaces: existence and algorithmic aspects, Advances in Operations Research. 2012(2012)843-486.

[15] Marino, G. and Xu, H.K., A general iterative method for nonexpansive mappings in Hilbert spaces, Math. Appl. 318(2006)43-52.

[16] Moradi, R. and Razani, A., Nonlinear iterative algorithms for quasi contraction mapping in modular space, Georgian Mathematics Journal, 23 (2016), No. 1, 105-119.

[17] Opial, Z., Weak convergence of the sequence of successive approximations for nonexpansive mappings, Bull. Am. Math. Soc. 73(4)(1967)595-597.

[18] Plubtieng, S. and Punpaeng, R., Fixed point solutions of variational inequalities for nonexpansive semigroups in Hilbert spaces, Math. Comput. Model. 48(2008) 279-286.

[19] Razani, A. and Bagherboum, M., Convergence and stability of Jungck-type iterative procedures in convex b-metric spaces, Fixed Point Theory and Applications, 2013:331 (2013),17 pages.

[20] Razani, A. and Yazdi, M., A new iterative method for nonexpansive mappings in Hilbert Spaces, Journal of Nonlinear and Optimization: Theory and Applications, 3 (2012), No.1, 85-92.

[21] Razani, A. and Yazdi, M., Viscosity approximation methods for a countable family of quasinonexpansive mappings, World Applied Sciences Journal, 17(12) (2012), 1618-1622.

[22] Razani, A. and Yazdi, M., A new iterative method for generalized equilibrium and fixed point problems of nonexpansive mappings, Bull. Malays. Math. Sci. Soc. (2) 35(4) (2012), $1049-1061$.

[23] Razani, A. and Yazdi, M., A new iterative method for a family of nonexpansive mappings, Mathematical Reports, 16(66), 1 (2014), 7-23.

[24] Rizvi, S. H., General Viscosity Implicit Midpoint Rule For Nonexpansive Mapping, 2016.

[25] Schneider, C., Analysis of the linearly implicit mid-point rule for differential-algebra equations, Electron. Trans. Numer. Anal., 1 (1993) 1-10.

[26] Shimizu, T. and Takahashi, W., Strong convergence to common fixed points of families of nonexpansive mappings, J. Math. Anal. Appl. 211(1997)71-83.

[27] Somalia, S. and Davulcua, S., Implicit midpoint rule and extrapolation to singularly perturbed boundary value problems, Int. J. Comput. Math., 75(1) (2000) 117-127.

[28] Somalia, S., Implicit midpoint rule to the nonlinear degenerate boundary value problems, Int. J. Comput. Math., 79(3) (2002) 327-332.

[29] Xu, H. K., Viscosity approximation method for nonexpansive semigroups, J. Math. Anal. Appl. 298(2004)279-291.

[30] Xu, H.K., Alghamdi, M.A. and Shahzad, N., The viscosity technique for the implicit mid point rule of nonexpansive mappings in Hilbert spaces, Fixed point Theory Appl., 41 (2015), 12 pages. 
Current address: M. Cheraghi: Department of Mathematics, Science and Research Branch, Islamic Azad university, Tehran, Iran

E-mail address: mcheraghi98@gmail.com

ORCID Address: http://orcid.org/0000-0003-4878-9250

Current address: M. Azhini: Department of Mathematics, Science and Research Branch, Islamic Azad university, Tehran, Iran

E-mail address: mahdi.azhini@gmail.com

ORCID Address: http://orcid.org/0000-0001-6390-0266

Current address: H.R. Sahebi (Corresponding author): Department of Mathematics, Ashtian Branch, Islamic Azad University, Ashtian, Iran.

E-mail address: sahebi@aiau.ac.ir

ORCID Address: http://orcid.org/0000-0002-1944-5670 